\title{
Study on Neutron-induced Background in the CRESST Experiment
}

H. Wulandari, F. von Feilitzsch, M. Huber, Th. Jagemann, J. Jochum, T. Lachenmaier, J.-C. Lanfranchi, W. Potzel, W. Rau, M. Stark, \& S. Waller

Physik Department E-15, Technische Universität München, James-Franckstr., D-85747 Garching, Germany

\begin{abstract}
CRESST II is an experiment for direct WIMP search, using cryogenic detectors. The ratio of the two signals (temperature rise and scintillation light) measured for each interaction is an excellent parameter for discrimination of the radioactive background. The main remaining background is the neutron flux present at the experimental site, since neutrons produce the same signals as WIMPs do. Based on Monte Carlo simulations the present work shows how neutrons from different origins affect CRESST and which measures have to be taken into account to reach the sensitivity goal.
\end{abstract}

\section{Sources of Neutron Background}

The flux of neutrons in the vicinity of CRESST (Jagemann et al. 2004) is dominated by low energy neutrons induced by radioactivity in the surrounding rock/concrete. In addition, a very small remaining impurity in the material used in the setup can induce significant background if higher sensitivity is to be aimed for.

Although the flux of high energy neutrons induced by muons in the rock is small compared to the total flux at the experimental site, this component can penetrate a neutron moderator, reach the setup and produce additional neutrons through spallations. Moreover, neutrons can be produced by muons in the experimental setup, particularly in the lead shield.

To investigate the contribution of each neutron source to the expected background rate in CRESST II, a study based on Monte Carlo simulations has been performed. For this purpose different Monte Carlo codes have been employed, namely MCNP4B (Briesmeister 1997), MCNPX (Waters 1999), FLUKA (Fasso 2001a) and MUSIC (Antonioli 1999).

\section{Neutron Background Rates Expected in CRESST II}

The contributions of different neutron sources to the count rate in the $15-25 \mathrm{keV}$ interval in a $\mathrm{CaWO}_{4}$ detector are shown in Table 1. In CRESST II, $60 \mathrm{GeV}$ WIMPs with a cross section as claimed by the DAMA experiment (Bernabei et al. 2000) would give $55 \mathrm{cts} / \mathrm{kg} / \mathrm{y}$ in the same energy range. This makes 
it difficult for CRESST II to check the DAMA evidence without a neutron moderator. The flux of low energy neutrons from the surrounding rock/concrete can be reduced effectively by a hydrogen-rich material like polyethylene. For the CRESST II setup a polyethylene shield (35-50 cm thick) is advisable. This will reduce the background count rate in the $\mathrm{CaWO}_{4}$ detector by up to three orders of magnitude. Then the background will be dominated by neutrons from other origins (see Table 1) and the sensitivity of the experiment for the WIMP-nucleon cross section would be limited to about $10^{-7} \mathrm{pb}$.

Table 1. Contributions of different neutron sources to the count rate at $15-25 \mathrm{keV}$ in a $\mathrm{CaWO}_{4}$ detector inside the CRESST setup.

\begin{tabular}{l|c}
\hline Neutron origin & $\begin{array}{c}\text { Count Rate } \\
\text { (cts } / \mathrm{kg} / \mathrm{y} \text { ) }\end{array}$ \\
\hline Low energy neutrons from the rock: (i) no moderator & 50 \\
(ii) $\mathrm{Pb} / \mathrm{Cu}+50 \mathrm{~cm} \mathrm{PE}$ & 0.04 \\
Low energy neutrons from fission of $0.1 \mathrm{ppb}{ }^{238} \mathrm{U}$ in the lead & 0.2 \\
High energy neutrons produced by muons in the rock & 0.3 \\
High energy neutrons produced by muons in the shields & 1 \\
\hline \hline
\end{tabular}

The remaining neutron flux with the neutron moderator installed is dominated by neutrons induced by muons in the lead shield. It is possible to suppress this neutron background by detecting the muons and rejecting muon coincident events. For CRESST II a muon veto system is planned in addition to a neutron moderator. This will enable CRESST II to reach the projected sensitivity of below $10^{-7} \mathrm{pb}$. The muon veto will be placed inside the polyethylene shield and will have an efficiency of more than $90 \%$.

However, the muon veto will reduce the neutron background only by a factor of three, unless high energy neutrons from the rock can be overcome. Neutrons scattered in more than one detector can be rejected as background, because WIMPs do not multiply-scatter. Therefore the neutron background will be further reduced and multiple scattering can also be engaged to determine the remaining single scatter neutron background. Such a simulation with an array of detectors will be done in the near future to investigate which sensitivity level can be reached by this technique.

\section{References}

Antonioli, P. et al. 1999, Phys. Lett. B, 471, 251

Bernabei, R. et al. 2000, ROM2F/01

Briesmeister, J.F. ed. 1997, MCNP-A General Monte Carlo N-Particle Code, Version 4B, LA-12625-M, Los Alamos National Laboratory

Fasso, A. et al. 2001a, in Proc. of the Monte Carlo 2000 Conf., eds. King, A. et al. (Berlin: Springer Verlag), p. 159 \& 955

Jagemann, Th. et al. 2004, these proceedings

Waters, L.S. 1999, MCNPX User's Manual - Version 2.1.5, Los Alamos National Laboratory Report, TPO-E83-G-UG-X-00001 